\title{
Yield and Ratooning Ability of Thirty Sugar Cane Genotypes
}

\author{
M. I. Masri" and M. M. M. Amein \\ Agronomy Department, Faculty of Agriculture, Cairo \\ Univeristy, Cairo Egypt.
}

\begin{abstract}
A $\mathrm{N}$ EXPERIMENT field was conducted at El- Mattana Agricultural Research Station, Luxor Governorate, to evaluate the yield and ratooning ability of 30 sugar cane genotypes (clones) along with the check cultivar G.T 54/9 during three different crop cycles; plant cane (PC), first ratoon (FR), and second ratoon (SR) crops during 2011/2013 harvesting seasons. A randomized complete block design with three replicates was used. Results indicated that the evaluated genotypes varied significantly $(P=0.05)$ within and among crop cycles for stalk length, stalk diameter, stalk density, stalk weight, stalks number, cane yield, Brix, sucrose content, purity, sugar recovery and sugar yield. The evaluated genotypes differed significantly in their ratooning ability (RA) for all studied traits. Over evaluated genotypes, means of stalk diameter, stalk weight, stalk density and cane yield in plant cane were higher than those in the first and second ratoon crops, while means of Brix, sucrose, purity and sugar recovery percentages in the second ratoon were higher than those in plant cane and first ratoon crops.

The relative influence of genotypic variance $\left(\delta^{2} \mathrm{~g}\right)$ in determining the phenotypic variance was primary to genotype by crop interaction variance $\left(\delta^{2} \mathrm{gc}\right)$ and error variance $\left(\delta^{2} \mathrm{e}\right)$ for all studied traits. Broad sense heritability $(\mathrm{H} \%)$ estimates were high for cane yield and its components as well as sugar yield, since it ranged from $82.53 \%$ for cane yield to $95.06 \%$ for stalk length. High genotypic coefficient of variation (GCV\%) estimates were for stalk weight ( $27.01 \%)$, stalk density $(39.36$ $\%)$ and stalk number (32.57\%), while low estimates were for stalk length and juice quality traits $(2.05 \% \leq \mathrm{GCV} \% \geq 7.99 \%)$. Genotypic variance $\left(\delta^{2} \mathrm{~g}\right)$ was primary to error variance $\left(\delta^{2} \mathrm{e}\right)$ for RA of cane yield and its components as well as sugar yield and juice quality traits. The highest heritability estimates of RA were for stalk weight $(98.10 \%)$, stalk density $(98.24 \%)$, stalk number $(99.46 \%)$ and sugar yield $(96.73 \%)$ with high estimates of genotypic coefficient of variation (GCV) for the same traits $(22.78 \%, 32.26 \%, 36.41 \%$ and $27.26 \%$, respectively).
\end{abstract}

Keywords: Saccharum spp. Yield performance, Ratooning ability, Broad-sense genetic variance, Genotype by crop interaction variance $\left(\delta^{2} \mathrm{gc}\right)$.

Sugarcane is a clonally propagated crop and in Egypt, it is typically harvested for plant cane and a number of ratoon crops. Planting operations and seed (stalk for

\footnotetext{
${ }^{\#}$ Corresponding author email": mimasri73@gmail.com
} 
vegetative propagation) costs constitute the largest input in sugarcane production (Salassi \& Giesler, 1995). Sugarcane yield decline was commonly observed in advanced ratoon crops and, hence, limits the economic production of sugarcane (Ricaud \& Arceneaux, 1986; Johnson et al., 1993 and Mirzawan \& Sugiyarta, 1999). The reasons for this decline are complex, but primarily related to diseases, insects and management practices (Shrivastava et al., 1992). Additionally, genotypes can vary substantially in their ratoon crop yields. Variation among sugarcane genotypes in respect of yield performance and ratooning ability (RA) for cane yield and its components has been reported by Tripathi et al. (1982), Chapman (1988), Milligan et al. (1990 a), Chapman et al. (1992), Jamil et al. (2007), El-Hinnawy \& Masri (2009 b), Arian et al. (2011) and Tahir et al. (2014) who found that at early selection stage of sugarcane, stalk diameter, and stalk weight were decreased in older crops, while stalks number, cane yield, juice quality traits, and sugar yield were increased in older crops. However, Orgeron et al. (2007) and El-Hinnawy \& Masri (2009 a) reported that at final selection stages, cane yield and sugar yield were decreased from plant cane to second ratoon crop, while stalks number was increased from plant cane to the first ratoon crop, while decreased from the first to second ratoon crop. Bhatnagar et al. (2003) reported that sugarcane clones varied in their ability to survive and produce a profitable ratoon crop. Since the ratooning behavior of a sugarcane variety is a function of genotype and environment interaction; a good ratooning genotype in one environment may not necessarily be a good ratooner in another environment. It is, therefore, necessary to identify genotypes with good ratooning ability for specific conditions. Olaoye (2005) reported that, genotypes with poor ratooning ability were characterized mostly by a sharp decline in cane yield especially between the plant cane and the first ratoon crop, whereas those with good RA had the highest yield decline between the first and second ratoon crops.

The clonal nature of sugarcane reproduction suggests broad-sense genetic estimates of variance and covariance are relevant genetic estimates for predictive use between clonal stages. Genetic variance estimates are usually applicable only to the specific population and range of tested environments (Falconer, 1989). Estimating genetic variances under a limited range of environmental conditions may lead to biased genetic variance estimates (Dudley \& Moll, 1969). Moreover, Kang et al. (1984) reported that sugarcane genetic variance estimates obtained from a single year and/or location would cause the genotypes by environment interaction variance (GE) estimates to be possibly biased or not estimable.

Reported genetic studies with sugarcane have used a wide range of populations and environments (Hogarth et al., 1981; Kang et al., 1983; Kang et al., 1984; El-Hinnawy \& Masri, 2009a and Jamoza et al., 2014). They reported estimates of heritability for sugarcane yield and its components to be relatively high. They also reported the least potential for selection gain existed for Brix and purity, followed by stalk length and stalk diameter; however, they found stalk weight and sucrose concentration to offer the largest potential for gain. While, Milligan et al. (1990 a) found stalks number and cane yield to offer the most potential.

Egypt. J. Agron . 37, No. 2 (2015) 
Ratooning ability can be enhanced by a direct selection of genotypes with high ratoon crop yields. Characters such as cane yield and its components; stalk length, stalk diameter, stalks number and stalk weight have been suggested as being indicative of better ratooning cultivars (Milligan et al., 1996); sucrose yield was also suggested because of its strong relationship to cane yield (Milligan et al., $1990 \mathrm{~b}$ and Masri et al., 2008).

The objectives of this study were (i) to investigate the ratooning ability and the yield performance of thirty sugarcane genotypes under three different crop cycles; plant cane (PC), first ratoon (FR), and second ratoon crops (SR), and (ii) to estimate broad-sense genetic and genotype by crop interaction (GC) variance components.

\section{Material and Methods}

The study was carried out at El- Mattana Agricultural Research Station, Luxor Governorate, to evaluate thirty sugarcane (Saccharum spp L.) clones along with the check cultivar GT 54/9 (represent more than 95\% of the planted sugarcane area in Egypt) for ratooning ability, as well as, yield and some of its attributes in plant cane, first and second ratoon crops during 2011, 2012 and 2013 harvest seasons, respectively. Sugarcane clones (Table 1) were selected from the line stage (the first clonal selection stage) and were grown in $5 \mathrm{~m} \mathrm{x} 3$ row plots. Distance between rows was $1.0 \mathrm{~m}$, thus plot area was $15 \mathrm{~m}^{2}$. A randomized complete block design with three replications was used. Planting was done during the second week of March 2010 season. Planting was achieved by placing twenty five 3-budded cane pieces in each row. Field was irrigated immediately right after planting and all other agronomic practices were carried out as recommended. Plant cane was allowed to ratoon (first and second ratoons). Harvest took place after 12 months from planting or harvest of plant cane or harvest of first ratoon. At harvest, data were recorded for the three crop years following planting; plant cane (PC), first ratoon (FR), and second ratoon (SR) as follows:

\section{Cane yield and its contributing traits}

A sample of twenty stalks from each plot was removed to measure stalk length, and stalk diameter.

1- Stalk length $(\mathrm{cm})$ was measured from soil surface to the visible dewlap.

2- Stalk diameter $(\mathrm{cm})$ was measured at midstalk with no reference to the bud groove.

3- Number of millable stalks/feddan (one feddan $=0.42$ ha) was calculated on a plot basis.

4- Stalk weight $(\mathrm{kg})$ was calculated by dividing cane yield per plot by number of stalks per plot.

5- Stalk density $\left(\mathrm{g} \mathrm{cm}^{-3}\right)$ was calculated as stalk weight per unit volume according to Milligan et al. (1990b), where volume $=$ length . $\pi$. adius $^{2}$.

6- Cane yield (ton/feddan) was calculated on plot basis. 
TABLE 1. Experimental bi-parental crosses and the selected 30 sugarcane clones used in this study.

\begin{tabular}{|c|c|c|c|c|c|}
\hline \multirow{2}{*}{ S. $\mathbf{N}$} & \multirow{2}{*}{ Clone } & \multicolumn{4}{|c|}{ Pedigree } \\
\hline & & Female & Origin & Male & Origin \\
\hline 1 & G $2008-23$ & \multirow{7}{*}{$\mathrm{CO} 842$} & \multirow{7}{*}{ India } & \multirow{7}{*}{ CP 34-38 } & \multirow{7}{*}{ U.S.A } \\
\hline 2 & G $2008-26$ & & & & \\
\hline 3 & G $2008-29$ & & & & \\
\hline 4 & G $2008-37$ & & & & \\
\hline 5 & G $2008-38$ & & & & \\
\hline 6 & G $2008-39$ & & & & \\
\hline 7 & G $2008-46$ & & & & \\
\hline 8 & G $2008-51$ & \multirow{6}{*}{ T 80-4897 } & \multirow{6}{*}{ Taiwan } & \multirow{6}{*}{ CP 79-318 } & \multirow{6}{*}{ U.S.A } \\
\hline 9 & G $2008-53$ & & & & \\
\hline 10 & G $2008-55$ & & & & \\
\hline 11 & G $2008-57$ & & & & \\
\hline 12 & G $2008-59$ & & & & \\
\hline 13 & G $2008-64$ & & & & \\
\hline 14 & G $2008-9$ & \multirow{5}{*}{$\mathrm{Ph} 6722$} & \multirow{5}{*}{ Philippine } & \multirow{5}{*}{ BO 18} & \multirow{5}{*}{ Barbados } \\
\hline 15 & G $2008-12$ & & & & \\
\hline 16 & G $2008-13$ & & & & \\
\hline 17 & G $2008-27$ & & & & \\
\hline 18 & G $2008-30$ & & & & \\
\hline 19 & G $2008-8$ & \multirow{4}{*}{$\mathrm{Ph} 8013$} & \multirow{4}{*}{ Philippine } & \multirow{4}{*}{ BO 41} & \multirow{4}{*}{ Barbados } \\
\hline 20 & G $2008-10$ & & & & \\
\hline 21 & G $2008-11$ & & & & \\
\hline 22 & G $2008-15$ & & & & \\
\hline 23 & G $2008-5$ & \multirow{3}{*}{ SP711-406 } & \multirow{3}{*}{ Brazil } & \multirow{3}{*}{ CP $67-412$} & \multirow{3}{*}{ U.S.A } \\
\hline 24 & G $2008-18$ & & & & \\
\hline 25 & G 2008 - 45 & & & & \\
\hline 26 & G $2008-54$ & \multirow{2}{*}{ T 75-6667 } & \multirow{2}{*}{ Taiwan } & \multirow{2}{*}{ T 82-4536 } & \multirow{2}{*}{ Taiwan } \\
\hline 27 & G $2008-63$ & & & & \\
\hline 28 & G $2008-7$ & SP 711-406 & Brazil & CO 842 & India \\
\hline 29 & G $2008-50$ & Т $82-4510$ & Taiwan & ROC 8 & Taiwan \\
\hline 30 & G $2008-61$ & T $70-3898$ & Taiwan & Т 81-107 & Taiwan \\
\hline 31 & GT 54-9 (Check) & & & & \\
\hline
\end{tabular}

Juice quality traits and sugar yield

The twenty stalk samples taken from each plot was crushed and juice was analyzed to determine the following traits:

1- Brix (percent soluble solids) was determined with a hydrometer.

2- Sucrose percentage of clarified juice was determined by using automated sacharimeter according to A.O.A.C. (1995).

Egypt. J. Agron. 37, No. 2 (2015) 
3- Purity was calculated as: [(Sucrose / Brix $)$ x 100].

4- Sugar recovery\% (rendment) was calculated according to the formula described by Yadav \& Sharma (1980) which is given below:

$\mathrm{SR}=[$ Sucrose \% $-0.4($ Brix - Sucrose \%) $]$ x 0.73

where: $0.4=$ each pound of non-sucrose solids in the juice will retain 0.4 of a pound of sucrose as outlined by Herbert (1973), and 0.73 is a correction factor for actual milling conditions in factories that depends on the overall mean cane fiber percentage during processing as outlined by Mathur (1997).

5- Sugar yield (ton/feddan) was estimated by multiplying net cane yield (ton/feddan) by sugar recovery percentage.

Ratooning ability (RA) is defined as: $\mathrm{RA}_{i}=100 \mathrm{SR}_{i} / \mathrm{PC}_{i}$ following Milligan et al. (1996);

where, RA of trait $i$ is defined as the ratio of second ratoon crop (SR) yield of trait $i$ to the plant cane yield (PC) of trait $i$ expressed as a percent.

Data were statistically analyzed using analysis of variance according to procedures outlined by Steel et al. (1997) using MSTAT-C computer package by Freed et al. (1989). Treatment mean comparisons were performed using least significant difference (LSD) at $5 \%$ level of probability. Genetic variance $\left(\delta^{2} \mathrm{~g}\right)$ and broad-sense heritability (H \%) were calculated according to Falconer (1989). Variance components were calculated by equating appropriate mean squares to their expectations and solving for the components. The full model that included crop effect and crop interaction effect was used for calculating genetic variance and heritability for yield and contributing traits:

$T_{i j k}=M+G_{i}+C_{j}+G_{i j}+R_{k(j)}+E_{i j k}$

where

$\mathrm{T}_{\mathrm{ijk}} \quad$ is the observation $\mathrm{k}$, in crop $\mathrm{j}$, of genotype $\mathrm{i}$;

$\mathrm{M}$ is the mean;

$\mathrm{G}_{\mathrm{i}} \quad$ is the genotype effect;

$\mathrm{C}_{\mathrm{j}} \quad$ is the crop effect;

$\mathrm{GC}_{\mathrm{ij}} \quad$ is the genotype $\mathrm{i}$ in crop $\mathrm{j}$;

$R_{k(j)}$ is the replication effect;

$\mathrm{E}_{\mathrm{ijk}} \quad$ is the residual.

Heritability estimate using variance components from the full model analysis were calculated as: $\mathrm{H}=\delta^{2} \mathrm{~g} /\left(\delta^{2} \mathrm{~g}+\delta^{2} \mathrm{gc} / \mathrm{c}+\delta^{2} \mathrm{e} / \mathrm{rc}\right)$

where, $\delta^{2} \mathrm{~g}, \delta^{2} \mathrm{e}$ and $\delta^{2} \mathrm{gc}$ refers to genotypic, error and genotype by crop interaction variance, respectively. The divisors $\mathrm{r}$ and $\mathrm{c}$ refers to number of replications and crops, respectively. The reduced model was used to estimate heritability for ratooning ability as: $\mathrm{H}=\delta^{2} \mathrm{~g} /\left(\delta^{2} \mathrm{~g}+\delta^{2} \mathrm{e} / \mathrm{r}\right)$.

Genetic coefficient of variation (GCV) provide a unitless measure of a trait's genetic variance relative to its mean and calculating as the following equation: $\mathrm{GCV} \%=(\delta \mathrm{g} /$ general mean $) \times 100$. 


\section{Results and Discussion}

All studied traits; stalk length, stalk diameter, stalk density, stalk weight, stalk numbers, cane yield, Brix, sucrose $\%$, purity $\%$, sugar recovery, and sugar yield were significantly $(\mathrm{P}=0.05)$ different among genotypes in plant cane $(\mathrm{PC})$, first ratoon (FR), and second ratoon (SR) crops (Tables 2, 3, 4, 5, 6 and 7). Sugar yield and all of its attributes were significantly affected by crop age. The genotype by crop age interaction was significant for all studied traits, indicating that genotype performance differed among the crop cycles. Milligan et al. (1990a), Orgeron et al. (2007) and El-Hinnawy \& Masri (2009a) reported that genotype by crop interaction was important for sugarcane yield and its component traits. The evaluated genotypes were significantly differed in their ratooning ability for all studied traits in agreement with the results obtained by Milligan et al. (1996), Olaoye (2005) and El-Hinnawy \& Masri (2009b).

Data presented in Table 2, revealed that stalk length of ten genotypes; $G$ 2008-23, G 2008-53, G 2008-57, G 2008- 9, G 2008-11, G 2008-18, G 200845, G 2008-54, G 2008-7 and G 2008-50 was increased with older crops, while stalk length of other genotypes either decreased with older crops or fluctuated among crops. On the other hand, average stalk length of the studied genotypes was increased in the first and second ratoon crops by $1.92 \%$ and $1.28 \%$ compared to plant cane crop. Stalk diameter of six genotypes; G 2008-55, G 2008- 64, G 2008-13, G 2008-15, G 2008-18 and G 2008- 63 was increased with older crops, while stalk diameter of other genotypes either decreased with older crops or fluctuated among crops.

Over all evaluated genotypes, stalk diameter of the studied genotypes was decreased in the first and second ratoon crops by $4.86 \%$ and $2.84 \%$ compared to plant cane crop. Ratooning ability estimates of about $65 \%$ (20 clones) and $48 \%$ (15 clones) of the evaluated genotypes for stalk length and stalk diameter, respectively, exceeded the unity, indicating that length and diameter of stalks were increased in the second ratoon crop compared to plant cane crop for the corresponding genotypes.

Data in Table 3, revealed that five genotypes; G 2008-29, G 2008- 39, G 2008-11, G 2008-50 and G 2008-61 recorded the heaviest mean stalk weight and significantly surpassed the commercial cultivar GT 54/9 during the three crop ages, but only two of them; G 2008-50 and G 2008- 61 were good ratooner genotypes. However, about $53 \%$ of the evaluated genotypes (16 clones) showed high ratooning ability for stalk weight and the highest value of RA was recorded by the clone G 2008- 64 (137.94\%) with significance differences with the check cultivar GT 54/9 (79.40\%). Three genotypes; G 2008- 11, G 2008- 54 and G $2008-61$ recorded the highest stalk density and significantly exceeded the check cultivar during the three crop cycles. Three genotypes; G 2008-55, G 2008-64 and G 2008- 63 significantly exceeded the check cultivar for stalk density during the first and second ratoon crops. The superiority of these genotypes for stalk density may be due to its low content of fiber and high content of juice. The Egypt. J. Agron. 37, No. 2 (2015) 
same clones (16 clones) that showed high RA for stalk weight recorded high RA for stalk density and the highest value of RA was recorded by the clone G 2008 $8(152.87 \%)$ with a significant difference with the check cultivar GT 54/9 $(73.23 \%)$.

TABLE 2. Mean performance and ratooning ability (RA\%) of thirty one sugarcane genotypes for stalk length and stalk diameter in plant cane (PC),first ratoon $(\mathrm{FR})$ and second ratoon $(\mathrm{SR})$ crops.

\begin{tabular}{|c|c|c|c|c|c|c|c|c|c|}
\hline \multirow{2}{*}{ S. $\mathbf{N}$} & \multirow{2}{*}{ Clone } & \multicolumn{4}{|c|}{ Stalk length $(\mathrm{cm})$} & \multicolumn{4}{|c|}{ Stalk diameter $(\mathrm{cm})$} \\
\hline & & PC & FR & SR & RA\% & PC & FR & SR & RA\% \\
\hline 1 & G 2008-23 & 263.83 & 270.00 & 271.67 & 102.98 & 2.16 & 1.97 & 1.63 & 75.80 \\
\hline 2 & G 2008-26 & 251.49 & 248.33 & 250.00 & 99.43 & 1.73 & 1.62 & 1.97 & 113.51 \\
\hline 3 & G 2008-29 & 305.00 & 295.00 & 291.67 & 95.66 & 2.53 & 2.42 & 2.37 & 93.44 \\
\hline 4 & G 2008-37 & 210.00 & 213.33 & 210.00 & 100.04 & 2.13 & 2.10 & 1.97 & 92.27 \\
\hline 5 & G 2008-38 & 230.00 & 235.00 & 238.33 & 103.67 & 2.37 & 2.07 & 2.00 & 84.64 \\
\hline 6 & G 2008-39 & 256.67 & 271.67 & 268.33 & 104.66 & 2.77 & 2.47 & 2.38 & 86.18 \\
\hline 7 & G 2008-46 & 241.67 & 253.33 & 226.67 & 93.87 & 2.37 & 2.20 & 2.07 & 87.36 \\
\hline 8 & G 2008-51 & 280.00 & 280.00 & 260.00 & 92.90 & 2.73 & 2.63 & 2.40 & 88.05 \\
\hline 9 & G 2008-53 & 246.67 & 258.33 & 261.67 & 106.15 & 2.65 & 2.45 & 2.75 & 103.77 \\
\hline 10 & G 2008-55 & 305.00 & 291.67 & 296.67 & 97.30 & 2.43 & 2.50 & 2.54 & 104.57 \\
\hline 11 & G 2008-57 & 243.33 & 248.33 & 256.67 & 105.54 & 2.60 & 2.43 & 2.63 & 101.28 \\
\hline 12 & G 2008-59 & 250.51 & 256.67 & 248.33 & 99.28 & 2.50 & 2.47 & 2.55 & 102.19 \\
\hline 13 & G 2008-64 & 288.33 & 296.67 & 295.00 & 102.37 & 2.57 & 2.63 & 2.70 & 105.23 \\
\hline 14 & G 2008-9 & 263.03 & 270.00 & 275.00 & 104.68 & 2.20 & 1.85 & 1.90 & 86.55 \\
\hline 15 & G 2008-12 & 267.42 & 280.00 & 273.33 & 102.27 & 1.93 & 1.92 & 2.12 & 109.52 \\
\hline 16 & G 2008-13 & 253.33 & 248.33 & 261.67 & 103.29 & 2.00 & 2.00 & 2.20 & 110.00 \\
\hline 17 & G 2008-27 & 280.00 & 275.00 & 280.00 & 100.02 & 2.20 & 1.77 & 2.04 & 92.88 \\
\hline 18 & G 2008-30 & 235.00 & 246.67 & 243.33 & 103.67 & 2.71 & 2.60 & 2.65 & 97.79 \\
\hline 19 & G 2008-8 & 242.60 & 288.33 & 270.00 & 111.46 & 2.40 & 2.37 & 2.73 & 113.89 \\
\hline 20 & G 2008-10 & 288.61 & 270.00 & 271.67 & 94.14 & 2.47 & 2.10 & 1.87 & 75.62 \\
\hline 21 & G 2008-11 & 265.00 & 288.33 & 291.67 & 110.10 & 2.92 & 2.77 & 2.73 & 93.72 \\
\hline 22 & G 2008-15 & 280.00 & 270.00 & 266.67 & 95.30 & 2.57 & 2.63 & 2.78 & 108.46 \\
\hline 23 & G 2008-5 & 267.01 & 263.33 & 248.33 & 93.04 & 2.40 & 2.20 & 2.17 & 90.47 \\
\hline 24 & G 2008-18 & 235.00 & 260.00 & 260.00 & 110.66 & 2.45 & 2.53 & 2.62 & 106.83 \\
\hline 25 & G 2008-45 & 246.67 & 246.67 & 253.33 & 102.78 & 2.60 & 2.50 & 2.63 & 101.33 \\
\hline 26 & G 2008-54 & 281.67 & 290.00 & 295.00 & 104.74 & 2.97 & 2.87 & 2.87 & 96.67 \\
\hline 27 & G 2008-63 & 310.00 & 301.67 & 295.00 & 95.17 & 2.77 & 2.80 & 2.82 & 101.85 \\
\hline 28 & G 2008-7 & 245.00 & 251.67 & 261.67 & 106.85 & 2.47 & 2.33 & 2.45 & 99.36 \\
\hline 29 & G 2008-50 & 268.53 & 285.00 & 288.33 & 107.38 & 2.60 & 2.45 & 2.62 & 100.61 \\
\hline 30 & G 2008-61 & 300.00 & 298.33 & 296.67 & 98.91 & 2.80 & 2.77 & 2.87 & 102.51 \\
\hline 31 & $\begin{array}{l}\text { GT 54-9 } \\
\text { (Check) }\end{array}$ & 295.00 & 301.67 & 295.00 & 100.03 & 2.73 & 2.58 & 2.52 & 92.11 \\
\hline \multicolumn{2}{|c|}{ Mean } & 264.40 & 269.46 & 267.80 & 101.56 & 2.47 & 2.35 & 2.40 & 97.37 \\
\hline \multicolumn{10}{|c|}{ L.S.D at $5 \%$} \\
\hline \multicolumn{2}{|c|}{ Genotypes (G) } & 11.70 & 11.73 & 8.20 & 5.13 & 0.10 & 0.12 & 0.12 & 5.89 \\
\hline \multicolumn{2}{|c|}{ Crop age ( C) } & \multicolumn{3}{|c|}{3.99} & & \multicolumn{3}{|c|}{0.63} & \\
\hline \multicolumn{2}{|c|}{$\mathrm{G} \times \mathrm{C}$} & & 10.53 & & & & 0.11 & & \\
\hline
\end{tabular}


TABLE 3. Mean performance and ratooning ability (RA\%) of thirty one sugarcane genotypes for stalk weight and stalk density in plant cane (PC),first ratoon (FR) and second ratoon (SR) crops.

\begin{tabular}{|c|c|c|c|c|c|c|c|c|c|}
\hline \multirow{2}{*}{ S. $\mathbf{N}$} & \multirow{2}{*}{ Clone } & \multicolumn{4}{|c|}{ Stalk weight (kg) } & \multicolumn{4}{|c|}{ Stalk density $\left(\mathrm{g} / \mathrm{cm}^{3}\right)$} \\
\hline & & PC & FR & SR & RA\% & PC & FR & SR & RA \% \\
\hline 1 & G 2008-23 & 0.66 & 0.52 & 0.34 & 51.12 & 0.60 & 0.43 & 0.24 & 40.05 \\
\hline 2 & G 2008-26 & 0.52 & 0.43 & 0.66 & 127.13 & 0.35 & 0.27 & 0.51 & 143.34 \\
\hline 3 & G 2008-29 & 1.28 & 1.05 & 0.97 & 75.74 & 1.56 & 1.18 & 1.05 & 67.68 \\
\hline 4 & G 2008-37 & 0.49 & 0.47 & 0.35 & 72.00 & 0.34 & 0.33 & 0.23 & 66.43 \\
\hline 5 & G 2008-38 & 0.69 & 0.53 & 0.44 & 64.46 & 0.59 & 0.41 & 0.33 & 56.61 \\
\hline 6 & G 2008-39 & 1.30 & 0.98 & 0.96 & 73.95 & 1.45 & 1.04 & 0.97 & 66.54 \\
\hline 7 & G 2008-46 & 0.77 & 0.68 & 0.46 & 60.20 & 0.69 & 0.59 & 0.34 & 49.63 \\
\hline 8 & G 2008-51 & 1.32 & 1.20 & 1.01 & 76.64 & 1.59 & 1.39 & 0.99 & 62.93 \\
\hline 9 & G 2008-53 & 1.07 & 0.95 & 1.28 & 119.51 & 1.10 & 0.95 & 1.44 & 131.67 \\
\hline 10 & G 2008-55 & 1.04 & 1.12 & 1.25 & 120.40 & 1.21 & 1.28 & 1.48 & 122.42 \\
\hline 11 & G 2008-57 & 1.02 & 0.85 & 1.18 & 116.56 & 1.01 & 0.81 & 1.26 & 124.75 \\
\hline 12 & G 2008-59 & 0.99 & 0.96 & 1.04 & 105.07 & 0.98 & 0.95 & 1.04 & 106.93 \\
\hline 13 & G 2008-64 & 0.98 & 1.21 & 1.35 & 137.94 & 1.14 & 1.48 & 1.69 & 148.43 \\
\hline 14 & G 2008-9 & 0.68 & 0.47 & 0.51 & 75.43 & 0.62 & 0.37 & 0.42 & 68.46 \\
\hline 15 & G 2008-12 & 0.58 & 0.58 & 0.65 & 110.95 & 0.47 & 0.49 & 0.59 & 124.16 \\
\hline 16 & G 2008-13 & 0.57 & 0.47 & 0.70 & 123.92 & 0.45 & 0.37 & 0.63 & 140.78 \\
\hline 17 & G 2008-27 & 0.70 & 0.54 & 0.68 & 96.65 & 0.68 & 0.41 & 0.61 & 89.75 \\
\hline 18 & G 2008-30 & 0.96 & 0.88 & 0.92 & 96.67 & 0.96 & 0.89 & 0.94 & 98.12 \\
\hline 19 & G 2008-8 & 0.93 & 0.97 & 1.12 & 120.62 & 0.85 & 1.04 & 1.30 & 152.87 \\
\hline 20 & G 2008-10 & 1.08 & 0.87 & 0.87 & 80.20 & 1.21 & 0.77 & 0.69 & 57.08 \\
\hline 21 & G 2008-11 & 1.47 & 1.38 & 1.36 & 92.60 & 1.79 & 1.73 & 1.70 & 95.69 \\
\hline 22 & G 2008-15 & 1.04 & 1.15 & 1.22 & 117.36 & 1.18 & 1.28 & 1.42 & 121.50 \\
\hline 23 & G 2008-5 & 0.90 & 0.81 & 0.80 & 88.93 & 0.91 & 0.74 & 0.67 & 75.26 \\
\hline 24 & G 2008-18 & 0.87 & 0.94 & 1.02 & 117.17 & 0.79 & 0.98 & 1.08 & 138.49 \\
\hline 25 & G 2008-45 & 0.89 & 0.83 & 0.99 & 111.33 & 0.90 & 0.80 & 1.04 & 116.01 \\
\hline 26 & G 2008-54 & 1.07 & 1.01 & 1.00 & 93.65 & 1.40 & 1.32 & 1.32 & 94.93 \\
\hline 27 & G 2008-63 & 0.91 & 0.97 & 1.15 & 125.98 & 1.22 & 1.29 & 1.49 & 122.04 \\
\hline 28 & G 2008-7 & 0.88 & 0.85 & 0.98 & 111.36 & 0.83 & 0.78 & 0.98 & 118.15 \\
\hline 29 & G 2008-50 & 1.15 & 1.09 & 1.19 & 104.07 & 1.26 & 1.20 & 1.41 & 112.45 \\
\hline 30 & G 2008-61 & 1.19 & 1.19 & 1.27 & 106.62 & 1.57 & 1.54 & 1.69 & 108.29 \\
\hline 31 & GT 54-9 (Check) & 1.02 & 0.90 & 0.81 & 79.40 & 1.29 & 1.10 & 0.94 & 73.23 \\
\hline \multicolumn{2}{|c|}{ Mean } & 0.94 & 0.87 & 0.92 & 98.50 & 1.00 & 0.91 & 0.98 & 99.83 \\
\hline \multicolumn{2}{|c|}{ L.S.D at $5 \%$} & & & & & & & & \\
\hline \multicolumn{2}{|c|}{ Genotypes (G) } & 0.05 & 0.07 & 0.05 & 8.83 & 0.12 & 0.12 & 0.07 & 12.18 \\
\hline \multicolumn{2}{|c|}{ Crop age (C) } & \multicolumn{3}{|c|}{0.02} & & \multicolumn{3}{|c|}{0.04} & \\
\hline \multicolumn{2}{|c|}{$\mathrm{G} \times \mathrm{C}$} & \multicolumn{3}{|c|}{0.07} & & \multicolumn{3}{|c|}{0.10} & \\
\hline
\end{tabular}

Egypt. J. Agron. 37, No. 2 (2015) 
TABLE 4. Mean performance and ratooning ability (RA\%) of thirty one sugarcane genotypes for stalks number and cane yield in plant cane (PC),first ratoon (FR) and second ratoon (SR) crops.

\begin{tabular}{|c|c|c|c|c|c|c|c|c|c|}
\hline \multirow{2}{*}{ S. $\mathbf{N}$} & \multirow{2}{*}{ Clone } & \multicolumn{4}{|c|}{ Stalks number $\times 10^{3}$} & \multicolumn{4}{|c|}{ Cane yield (ton/feddan*) } \\
\hline & & $\mathbf{P C}$ & $\mathbf{F R}$ & SR & RA\% & $\mathbf{P C}$ & FR & SR & RA\% \\
\hline 1 & G 2008-23 & 54.53 & 58.54 & 75.78 & 139.16 & 36.09 & 30.48 & 25.48 & 70.75 \\
\hline 2 & G 2008-26 & 73.22 & 81.82 & 64.34 & 87.88 & 38.31 & 35.46 & 42.47 & 111.32 \\
\hline 3 & G 2008-29 & 33.20 & 44.47 & 49.76 & 149.85 & 42.59 & 46.79 & 48.32 & 113.47 \\
\hline 4 & G 2008-37 & 68.34 & 75.17 & 78.54 & 114.92 & 33.18 & 35.65 & 27.46 & 82.77 \\
\hline 5 & G 2008-38 & 73.45 & 83.59 & 89.80 & 122.28 & 50.32 & 44.32 & 39.61 & 78.83 \\
\hline 6 & G 2008-39 & 26.01 & 31.45 & 30.51 & 117.32 & 33.84 & 30.86 & 29.33 & 86.77 \\
\hline 7 & G 2008-46 & 54.89 & 65.29 & 67.91 & 123.77 & 42.29 & 44.09 & 31.47 & 74.46 \\
\hline 8 & G 2008-51 & 35.44 & 37.39 & 40.64 & 114.72 & 46.90 & 44.88 & 41.19 & 87.87 \\
\hline 9 & G 2008-53 & 40.49 & 44.45 & 36.56 & 90.29 & 43.29 & 42.41 & 46.64 & 107.96 \\
\hline 10 & G 2008-55 & 40.70 & 33.68 & 22.86 & 56.14 & 42.33 & 37.66 & 28.54 & 67.55 \\
\hline 11 & G 2008-57 & 44.39 & 53.00 & 31.14 & 70.20 & 45.09 & 45.13 & 36.84 & 81.94 \\
\hline 12 & G 2008-59 & 55.01 & 58.87 & 53.43 & 97.13 & 54.64 & 56.49 & 55.73 & 102.02 \\
\hline 13 & G 2008-64 & 43.22 & 34.18 & 24.51 & 56.84 & 42.33 & 41.24 & 33.10 & 78.27 \\
\hline 14 & G 2008-9 & 65.22 & 77.70 & 76.05 & 116.73 & 44.41 & 36.78 & 39.02 & 87.89 \\
\hline 15 & G 2008-12 & 70.10 & 70.66 & 53.87 & 76.88 & 40.88 & 40.93 & 34.83 & 85.20 \\
\hline 16 & G 2008-13 & 62.63 & 85.83 & 54.31 & 86.75 & 35.55 & 40.03 & 38.18 & 107.52 \\
\hline 17 & G 2008-27 & 58.03 & 76.78 & 61.44 & 105.95 & 40.64 & 41.45 & 41.57 & 102.35 \\
\hline 18 & G 2008-30 & 31.88 & 37.42 & 34.29 & 108.15 & 30.41 & 32.85 & 31.68 & 104.29 \\
\hline 19 & G 2008-8 & 31.17 & 26.53 & 16.10 & 51.80 & 28.96 & 25.60 & 18.06 & 62.61 \\
\hline 20 & G 2008-10 & 35.62 & 55.86 & 56.98 & 160.16 & 38.50 & 48.43 & 49.38 & 128.29 \\
\hline 21 & G 2008-11 & 24.52 & 33.76 & 37.34 & 152.93 & 35.98 & 46.53 & 50.77 & 141.20 \\
\hline 22 & G 2008-15 & 37.18 & 22.35 & 18.57 & 49.95 & 38.75 & 25.73 & 22.71 & 58.74 \\
\hline 23 & G 2008-5 & 47.26 & 56.46 & 59.16 & 125.31 & 42.56 & 45.72 & 47.31 & 111.17 \\
\hline 24 & G 2008-18 & 44.38 & 36.29 & 23.08 & 52.09 & 38.54 & 34.15 & 23.46 & 60.98 \\
\hline 25 & G 2008-45 & 46.33 & 53.61 & 25.77 & 55.68 & 41.21 & 44.63 & 25.51 & 62.01 \\
\hline 26 & G 2008-54 & 42.75 & 43.20 & 43.83 & 102.54 & 45.51 & 43.60 & 43.68 & 96.01 \\
\hline 27 & G 2008-63 & 45.98 & 38.12 & 26.55 & 57.97 & 41.78 & 36.70 & 30.39 & 72.95 \\
\hline 28 & G 2008-7 & 38.98 & 40.02 & 19.88 & 51.08 & 34.18 & 33.86 & 19.38 & 56.89 \\
\hline 29 & G 2008-50 & 44.23 & 44.81 & 21.00 & 47.51 & 50.58 & 48.87 & 25.01 & 49.45 \\
\hline 30 & G 2008-61 & 44.18 & 40.62 & 29.58 & 67.12 & 52.45 & 48.32 & 37.48 & 71.45 \\
\hline 31 & GT 54-9 (Check) & 48.91 & 56.74 & 62.22 & 127.28 & 49.70 & 51.07 & 50.18 & 100.99 \\
\hline \multicolumn{2}{|r|}{ Mean } & 47.17 & 51.57 & 44.70 & 94.72 & 41.35 & 40.67 & 35.96 & 87.23 \\
\hline \multicolumn{10}{|c|}{ L.S.D at $5 \%$} \\
\hline \multicolumn{2}{|r|}{ Genotypes (G) } & 2.57 & 3.21 & 2.19 & 7.16 & 2.82 & 3.65 & 2.20 & 7.86 \\
\hline \multicolumn{2}{|r|}{ Crop age ( C) } & & 1.25 & & & \multicolumn{3}{|c|}{0.53} & \\
\hline \multicolumn{2}{|r|}{$\mathrm{G} \times \mathrm{C}$} & & 2.65 & & & \multicolumn{4}{|c|}{2.91} \\
\hline
\end{tabular}

- One feddane $=0.42$ ha 
TABLE 5. Mean performance and ratooning ability (RA\%) of thirty one sugarcane genotypes for brix and sucrose percentages in plant cane (PC), first ratoon (FR) and second ratoon (SR) crops.

\begin{tabular}{|c|c|c|c|c|c|c|c|c|c|}
\hline \multirow{2}{*}{ S. $\mathbf{N}$} & \multirow{2}{*}{ Clone } & \multicolumn{4}{|c|}{ Brix \% } & \multicolumn{4}{|c|}{ Sucrose $\%$} \\
\hline & & PC & FR & SR & RA\% & PC & FR & SR & RA\% \\
\hline 1 & G 2008-23 & 21.04 & 21.67 & 21.95 & 104.32 & 17.01 & 17.82 & 18.08 & 106.33 \\
\hline 2 & G 2008-26 & 20.25 & 21.15 & 21.82 & 107.77 & 16.11 & 16.97 & 17.83 & 110.68 \\
\hline 3 & G 2008-29 & 21.07 & 21.80 & 22.33 & 106.04 & 17.00 & 17.87 & 18.33 & 107.85 \\
\hline 4 & G 2008-37 & 20.16 & 20.61 & 20.76 & 103.07 & 16.24 & 16.67 & 16.65 & 102.63 \\
\hline 5 & G 2008-38 & 21.25 & 21.42 & 22.05 & 103.78 & 17.30 & 17.74 & 18.32 & 105.85 \\
\hline 6 & G 2008-39 & 20.85 & 21.44 & 21.67 & 104.03 & 16.15 & 16.84 & 17.37 & 107.58 \\
\hline 7 & G 2008-46 & 21.77 & 22.20 & 22.40 & 103.02 & 17.57 & 18.00 & 18.40 & 104.79 \\
\hline 8 & G 2008-51 & 19.81 & 20.21 & 21.03 & 106.15 & 16.25 & 17.05 & 17.29 & 106.45 \\
\hline 9 & G 2008-53 & 21.59 & 22.40 & 22.68 & 105.08 & 17.78 & 18.57 & 18.81 & 105.81 \\
\hline 10 & G 2008-55 & 20.96 & 21.09 & 21.95 & 104.80 & 16.65 & 16.70 & 17.52 & 105.45 \\
\hline 11 & G 2008-57 & 20.89 & 21.55 & 21.67 & 103.77 & 16.95 & 16.89 & 17.94 & 106.26 \\
\hline 12 & G 2008-59 & 21.52 & 22.71 & 23.01 & 106.92 & 16.83 & 18.53 & 17.88 & 106.58 \\
\hline 13 & G 2008-64 & 22.83 & 20.15 & 21.02 & 92.04 & 18.74 & 16.69 & 16.01 & 85.64 \\
\hline 14 & G 2008-9 & 22.08 & 20.84 & 22.30 & 101.02 & 16.98 & 16.58 & 18.73 & 110.52 \\
\hline 15 & G 2008-12 & 18.94 & 21.99 & 23.20 & 122.68 & 14.61 & 17.28 & 17.37 & 118.85 \\
\hline 16 & G 2008-13 & 20.69 & 20.87 & 21.67 & 105.03 & 14.99 & 15.93 & 17.22 & 115.18 \\
\hline 17 & G 2008-27 & 19.92 & 21.43 & 22.57 & 113.34 & 15.95 & 16.80 & 17.19 & 107.87 \\
\hline 18 & G 2008-30 & 20.37 & 23.20 & 21.95 & 107.91 & 16.83 & 18.64 & 18.38 & 109.37 \\
\hline 19 & G 2008-8 & 21.38 & 21.55 & 21.20 & 99.18 & 16.56 & 15.78 & 18.50 & 111.80 \\
\hline 20 & G 2008-10 & 20.23 & 22.73 & 21.67 & 107.12 & 16.12 & 18.42 & 18.06 & 112.02 \\
\hline 21 & G 2008-11 & 18.78 & 19.94 & 20.67 & 110.10 & 14.63 & 15.83 & 16.47 & 112.58 \\
\hline 22 & G 2008-15 & 20.47 & 21.20 & 22.19 & 108.41 & 16.54 & 17.17 & 18.08 & 109.31 \\
\hline 23 & G 2008-5 & 20.69 & 21.25 & 21.78 & 105.40 & 16.12 & 16.93 & 17.63 & 109.50 \\
\hline 24 & G 2008-18 & 19.92 & 20.95 & 21.61 & 108.48 & 15.15 & 16.25 & 17.01 & 112.31 \\
\hline 25 & G 2008-45 & 19.91 & 20.78 & 20.70 & 103.98 & 15.20 & 16.21 & 16.60 & 109.28 \\
\hline 26 & G 2008-54 & 21.09 & 21.46 & 21.52 & 102.07 & 17.67 & 18.00 & 17.70 & 100.21 \\
\hline 27 & G 2008-63 & 20.09 & 21.80 & 21.95 & 109.24 & 16.51 & 17.97 & 18.05 & 109.32 \\
\hline 28 & G 2008-7 & 20.82 & 22.07 & 22.29 & 107.04 & 17.15 & 18.33 & 18.60 & 108.45 \\
\hline 29 & G 2008-50 & 21.62 & 22.00 & 22.07 & 102.10 & 17.73 & 18.13 & 18.33 & 103.42 \\
\hline 30 & G 2008-61 & 21.13 & 21.34 & 21.83 & 103.34 & 17.58 & 17.95 & 18.42 & 104.81 \\
\hline 31 & $\begin{array}{l}\text { GT 54-9 } \\
\text { (Check) }\end{array}$ & 21.73 & 22.40 & 22.62 & 104.09 & 18.09 & 18.40 & 18.58 & 102.72 \\
\hline \multicolumn{2}{|r|}{ Mean } & 20.77 & 21.49 & 21.88 & 105.53 & 16.61 & 17.32 & 17.79 & 107.40 \\
\hline \multicolumn{10}{|c|}{ L.S.D at $5 \%$} \\
\hline \multicolumn{2}{|c|}{ Genotypes (G) } & 0.77 & 0.41 & 0.89 & 5.93 & 0.93 & 0.42 & 0.63 & 7.35 \\
\hline \multicolumn{2}{|c|}{ Crop age (C) } & \multicolumn{3}{|c|}{0.29} & & \multicolumn{3}{|c|}{0.16} & \\
\hline \multicolumn{2}{|c|}{$\mathrm{G} \times \mathrm{C}$} & \multicolumn{3}{|c|}{0.71} & & \multicolumn{3}{|c|}{0.68} & \\
\hline
\end{tabular}

Egypt. J. Agron . 37, No. 2 (2015) 
TABLE 6. Mean performance and ratooning ability (RA\%) of thirty one sugarcane genotypes for purity and sugar recovery percentages in plant cane (PC), first ratoon (FR) and second ratoon (SR) crops.

\begin{tabular}{|c|c|c|c|c|c|c|c|c|c|}
\hline \multirow{2}{*}{ S. $\mathbf{N}$} & \multirow{2}{*}{ Clone } & \multicolumn{4}{|c|}{ Purity \% } & \multicolumn{4}{|c|}{ Sugar recovery \% } \\
\hline & & PC & FR & SR & RA\% & $\overline{P C}$ & FR & SR & RA\% \\
\hline 1 & G 2008-23 & 80.83 & 82.28 & 82.39 & 101.92 & 11.24 & 11.89 & 12.07 & 107.42 \\
\hline 2 & G 2008-26 & 79.56 & 80.21 & 81.72 & 102.71 & 10.55 & 11.16 & 11.85 & 112.33 \\
\hline 3 & G 2008-29 & 80.71 & 81.96 & 82.09 & 101.71 & 11.22 & 11.89 & 12.22 & 108.85 \\
\hline 4 & G 2008-37 & 80.52 & 80.85 & 80.20 & 99.59 & 10.71 & 11.01 & 10.95 & 102.38 \\
\hline 5 & G 2008-38 & 81.44 & 82.85 & 83.07 & 101.99 & 11.48 & 11.88 & 12.28 & 106.97 \\
\hline 6 & G 2008-39 & 77.50 & 78.53 & 80.15 & 103.53 & 10.41 & 10.95 & 11.42 & 109.75 \\
\hline 7 & G 2008-46 & 80.72 & 81.08 & 82.15 & 101.77 & 11.60 & 11.91 & 12.26 & 105.76 \\
\hline 8 & G 2008-51 & 82.00 & 84.35 & 82.24 & 100.30 & 10.82 & 11.52 & 11.53 & 106.61 \\
\hline 9 & G 2008-53 & 82.38 & 82.90 & 82.95 & 100.69 & 11.87 & 12.43 & 12.60 & 106.20 \\
\hline 10 & G 2008-55 & 79.52 & 79.20 & 79.82 & 100.79 & 10.90 & 10.91 & 11.50 & 106.19 \\
\hline 11 & G 2008-57 & 81.15 & 78.35 & 82.90 & 102.41 & 11.23 & 10.96 & 12.00 & 107.86 \\
\hline 12 & G 2008-59 & 78.24 & 81.60 & 77.71 & 99.64 & 10.92 & 12.31 & 11.55 & 106.70 \\
\hline 13 & G 2008-64 & 82.05 & 82.83 & 76.28 & 93.10 & 12.49 & 11.18 & 10.23 & 82.32 \\
\hline 14 & G 2008-9 & 77.03 & 79.52 & 84.04 & 109.45 & 10.91 & 10.85 & 12.63 & 116.53 \\
\hline 15 & G 2008-12 & 77.19 & 78.58 & 74.88 & 97.06 & 9.40 & 11.24 & 10.97 & 116.67 \\
\hline 16 & G 2008-13 & 72.45 & 76.30 & 79.59 & 109.80 & 9.28 & 10.18 & 11.27 & 121.76 \\
\hline 17 & G 2008-27 & 80.09 & 78.40 & 76.28 & 95.39 & 10.48 & 10.91 & 10.98 & 104.98 \\
\hline 18 & G 2008-30 & 82.60 & 80.35 & 83.80 & 101.42 & 11.25 & 12.28 & 12.37 & 110.13 \\
\hline 19 & G 2008-8 & 77.43 & 73.22 & 87.32 & 112.76 & 10.68 & 9.84 & 12.72 & 119.18 \\
\hline 20 & G 2008-10 & 79.67 & 81.04 & 83.41 & 104.69 & 10.57 & 12.19 & 12.12 & 114.76 \\
\hline 21 & G 2008-11 & 77.91 & 79.41 & 79.67 & 102.27 & 9.46 & 10.36 & 10.79 & 114.04 \\
\hline 22 & G 2008-15 & 80.83 & 81.01 & 81.47 & 100.84 & 10.93 & 11.36 & 12.00 & 109.85 \\
\hline 23 & G 2008-5 & 77.93 & 79.69 & 80.95 & 103.87 & 10.44 & 11.10 & 11.66 & 111.87 \\
\hline 24 & G 2008-18 & 76.05 & 77.56 & 78.73 & 103.54 & 9.67 & 10.49 & 11.08 & 114.63 \\
\hline 25 & G 2008-45 & 76.35 & 78.00 & 80.18 & 105.09 & 9.72 & 10.50 & 10.92 & 112.54 \\
\hline 26 & G 2008-54 & 83.77 & 83.88 & 82.25 & 98.19 & 11.90 & 12.13 & 11.81 & 99.25 \\
\hline 27 & G 2008-63 & 82.17 & 82.43 & 82.22 & 100.06 & 11.01 & 12.00 & 12.03 & 109.36 \\
\hline 28 & G 2008-7 & 82.38 & 83.09 & 83.46 & 101.33 & 11.45 & 12.29 & 12.50 & 109.20 \\
\hline 29 & G 2008-50 & 82.00 & 82.43 & 83.08 & 101.31 & 11.81 & 12.11 & 12.29 & 104.12 \\
\hline 30 & G 2008-61 & 83.21 & 84.14 & 84.39 & 101.44 & 11.79 & 12.11 & 12.45 & 105.61 \\
\hline 31 & GT 54-9 (Check) & 83.26 & 82.15 & 82.16 & 98.68 & 12.15 & 12.27 & 12.39 & 102.00 \\
\hline \multicolumn{2}{|c|}{ Mean } & 79.97 & 80.59 & 81.34 & 101.85 & 10.91 & 11.43 & 11.79 & 108.57 \\
\hline \multicolumn{10}{|c|}{ L.S.D at $5 \%$} \\
\hline \multicolumn{2}{|c|}{ Genotypes (G) } & 3.62 & 1.52 & 3.55 & 5.97 & 0.84 & 0.36 & 0.60 & 10.06 \\
\hline \multicolumn{2}{|c|}{ Crop age ( C) } & \multicolumn{3}{|c|}{0.72} & & \multicolumn{3}{|c|}{0.19} & \\
\hline \multicolumn{2}{|c|}{$\mathrm{G} \times \mathrm{C}$} & \multicolumn{3}{|c|}{3.02} & & \multicolumn{3}{|c|}{0.62} & \\
\hline
\end{tabular}


TABLE 7. Mean performance and ratooning ability (RA\%) of thirty one sugarcane genotypes for purity and sugar recovery percentages in plant cane (PC), first ratoon (FR) and second ratoon (SR) crops.

\begin{tabular}{|c|c|c|c|c|c|}
\hline \multirow{2}{*}{ S. $\mathbf{N}$} & \multirow{2}{*}{ Clone } & \multicolumn{4}{|c|}{ Sugar yield (ton/feddan) } \\
\hline & & PC & FR & SR & RA\% \\
\hline 1 & G 2008-23 & 4.06 & 3.63 & 3.07 & 75.99 \\
\hline 2 & G 2008-26 & 4.04 & 3.96 & 5.04 & 124.91 \\
\hline 3 & G 2008-29 & 4.78 & 5.57 & 5.90 & 123.51 \\
\hline 4 & G 2008-37 & 3.55 & 3.93 & 3.01 & 84.85 \\
\hline 5 & G 2008-38 & 5.78 & 5.26 & 4.86 & 84.32 \\
\hline 6 & G 2008-39 & 3.53 & 3.38 & 3.35 & 95.30 \\
\hline 7 & G 2008-46 & 4.91 & 5.25 & 3.86 & 78.71 \\
\hline 8 & G 2008-51 & 5.07 & 5.17 & 4.75 & 93.68 \\
\hline 9 & G 2008-53 & 5.14 & 5.28 & 5.88 & 114.71 \\
\hline 10 & G 2008-55 & 4.61 & 4.11 & 3.29 & 71.91 \\
\hline 11 & G 2008-57 & 5.08 & 4.95 & 4.43 & 89.20 \\
\hline 12 & G 2008-59 & 5.96 & 6.96 & 6.44 & 108.95 \\
\hline 13 & G 2008-64 & 5.28 & 4.61 & 3.39 & 64.47 \\
\hline 14 & G 2008-9 & 4.84 & 3.99 & 4.93 & 102.30 \\
\hline 15 & G 2008-12 & 3.84 & 4.60 & 3.82 & 99.38 \\
\hline 16 & G 2008-13 & 3.30 & 4.08 & 4.30 & 130.92 \\
\hline 17 & G 2008-27 & 4.26 & 4.52 & 4.56 & 107.32 \\
\hline 18 & G 2008-30 & 3.43 & 4.03 & 3.92 & 115.10 \\
\hline 19 & G 2008-8 & 3.09 & 2.52 & 2.30 & 74.73 \\
\hline 20 & G 2008-10 & 4.07 & 5.90 & 5.99 & 147.25 \\
\hline 21 & G 2008-11 & 3.40 & 4.82 & 5.48 & 161.04 \\
\hline 22 & G 2008-15 & 4.24 & 2.92 & 2.72 & 64.32 \\
\hline 23 & G 2008-5 & 4.44 & 5.08 & 5.52 & 124.60 \\
\hline 24 & G 2008-18 & 3.72 & 3.58 & 2.60 & 69.86 \\
\hline 25 & G 2008-45 & 4.02 & 4.69 & 2.79 & 69.84 \\
\hline 26 & G 2008-54 & 5.42 & 5.29 & 5.16 & 95.34 \\
\hline 27 & G 2008-63 & 4.60 & 4.41 & 3.66 & 79.68 \\
\hline 28 & G 2008-7 & 3.91 & 4.16 & 2.42 & 62.17 \\
\hline 29 & G 2008-50 & 5.97 & 5.92 & 3.07 & 51.45 \\
\hline 30 & G 2008-61 & 6.18 & 5.85 & 4.67 & 75.49 \\
\hline 31 & GT 54-9 (Check) & 6.04 & 6.26 & 6.22 & 102.99 \\
\hline \multicolumn{2}{|c|}{ Mean } & 4.53 & 4.67 & 4.24 & 94.98 \\
\hline \multicolumn{6}{|c|}{ L.S.D at $5 \%$} \\
\hline \multicolumn{2}{|c|}{ Genotypes (G) } & 0.52 & 0.44 & 0.35 & 13.48 \\
\hline \multicolumn{2}{|c|}{ Crop age ( C) } & \multicolumn{3}{|l|}{0.09} & \\
\hline \multicolumn{2}{|c|}{$\mathrm{G} \times \mathrm{C}$} & \multicolumn{3}{|l|}{0.44} & \\
\hline
\end{tabular}

Egypt. J. Agron. 37, No. 2 (2015) 
Among the evaluated genotypes, stalks number of 11 genotypes along with the check cultivar GT 54/9 (Table 4) was increased with the older crops, while stalk number of other genotypes either decreased with older crops or fluctuated among crops. Four genotypes (G 2008-37, G 2008-37, G 2008-46 and G 20089) of that showed an increase in stalks number with the older crops recorded the highest stalks number and significantly surpassed the check cultivar GT 54/9 during the three crop cycles, as well as, its high ratooning ability (114.92, $122.28,123.77$ and $116.73 \%$, respectively). However, the highest RA value for stalks number was recorded by the genotype G 2008-10 (160.16\%) followed by the genotypes G 2008-11(152.93\%), G2008- 29 (149.85\%) and G2008-23 $(139.16 \%)$ that significantly exceeded the check cultivar GT 54/9 (127.28\%). Cane yield of five genotypes; G 2008-29, G 2008-27, G 2008-10, G 2008-11 and $G$ 2008-5 was increased with older crops, therefore its ratooning ability value exceeded the unity (Table 4). Three genotypes; G 2008- 59, G 2008- 30 and G 2008- 54, as well as, the check cultivar GT 54/9 showed stability (consistency) in their performance for cane yield across the three crop cycles, since its yield did not differ significantly from plant cane up to the second ratoon crop, therefore its ratooning ability value nearly equal the unity. Among the evaluated genotypes, only one of them; G 2008-59 significantly surpassed the check cultivar in cane yield during the three crop cycles $(54.64,56.49$ and 55.73 ton/feddan vs. 49.70, 51.07 and 50.18 ton/fed in plant cane, first ratoon and second ratoon crops, respectively). The highest RA value $(141.20 \%)$ for cane yield was recorded by the genotype G 2008-11, indicating the superiority of cane yield in second ratoon crop over plant cane crop, while the lowest RA value $(49.45 \%)$ for cane yield was recorded by the genotype G 2008-50, indicating the high reduction in yield in the second ratoon crop. Over all evaluated genotypes, cane yield was decreased in the first and second ratoon crops by $1.64 \%$ and $13.03 \%$, respectively, compared to plant cane crop with no significant difference between plant cane and second ratoon crops. It is worth mentioning that the genotypes that show either increasing or consistent in their yielding performance across crops with high mean yield over crops such as G 2008-29 (45.90 ton/fed ), G 2008- 59 (55.62 ton/feddan), G 2008-27(41.22 ton/feddan), G 2008-10( 45.44 ton/feddan), G 2008-11(44.43 ton/feddan), G 2008-5(45.20 ton/feddan) and G 2008-54(44.26 ton/feddan) could be advanced to the next stage of selection aiming to saving costs of replanting of new sugar cane fields and increase the economic production of sugarcane in the future.

The previous results indicated that over evaluated genotypes, means of stalk diameter, stalk weight, stalk density and cane yield in plant cane were higher than those in the first and second ratoon crops. Similar results were reported by Milligan et al. (1990a) and El-Hinnawy \& Masri (2009a). Changes in RA and ratoon crop yields are usually, but not necessarily, related (Chapman et al., 1992). RA of cane yield and yield of cane (Table 4) indicated that an increase in ratoon yields may not increase the RA, but it would likely increase the number of economically productive ratoon crops such as for G 2008-59 and G 2008-27 genotypes, in which yield of the first genotype is greater than yield of the second genotype in both plant cane and second ratoon crops, yet they have similar RA. 
Data in Tables 5, 6, 7 revealed that the evaluated genotypes varied significantly within and among crop cycles for total soluble solids (Brix), sucrose percentage, juice purity, sugar recovery and sugar yield. Over studied genotypes, crop age had a significant effect on juice quality traits in agreement with the results obtained by Milligan et al. (1990a). In contrast, El- Hinnawy \& Masri (2009a) found that crop age had no significant effect on juice quality traits of sugarcane at advanced stage of selection. Juice quality traits in terms of Brix, sucrose, purity and sugar recovery percentages of most evaluated genotypes beside the check cultivar GT 54/9 (Tables 5, 6) were slightly increased or showed consistency with the older crops. In general, over evaluated genotypes, mean values of juice quality traits increased from plant cane to second ratoon crop. Over crops, the mean values of sucrose and sugar recovery percentages of the genotypes; G 2008-7 (18.03 and 12.08\%), G 2008-50 (18.06 and 12.07\%) and G 2008-61 (17.98 and 12.12\%) were nearly the same as the check cultivar GT 54/9 (18.36 and 12.27\%). Although the evaluated genotypes differ significantly in their ratooning ability for juice quality traits, the RA of Brix value, sucrose content, juice purity, and sugar recovery indicated little change between plant cane and second ratoon crop for most evaluated genotypes are in agreement with the results reported by El- Hinnawy \& Masri (2009b). Chapman (1988) reported that older crops tend to mature earlier than younger crops, but final sucrose concentration and its components, Brix, sucrose content, Juice purity and sugar recovery are generally not affected by crop age.

Sugar yield of most evaluated genotypes nearly followed the same trends as in cane yield. Sugar yield of seven genotypes; G 2008-29, G 2008-53, G 200813, G 2008-27, G 2008-10, G 2008-11 and G 2008-5 was increased with older crops with an average yield of 5.41, 5.43, 3.90, 4.45, 5.32, 4.57 and 5.01 ton/feddan over the three crop cycles, respectively. Therefore, the ratooning ability value of these genotypes exceeded the unity (Table 7). Of the previous genotypes; sugar yield in the second ratoon crop of G 2008-29 (5.90 ton), G 2008-53 (5.88 ton) and G 2008-10 (5.99 ton) did not differ significantly from the yield of the check cultivar (6.22 ton), while its yield in plant cane and first ratoon crops was significantly lower than the check cultivar and this explains high RA value of these genotypes as compared to that of the check cultivar. Sugar yield of the genotype G 2008- 50 in plant cane ( 5.97 ton) and first ratoon (5.92 ton ) was at bar as the check cultivar (6.04, and 6.26 ton in plant cane and first ratoon, respectively), thereafter its yield significantly decreased with the older crop (3.07 ton). Among the evaluated genotypes, only one of them; $G$ 2008-59 significantly surpassed the check cultivar in sugar yield during the first ratoon crop (6.96 ton), while its yield in plant cane (5.96 ton) and second ratoon crop (6.44 ton) did not differ significantly with yield of the check cultivar GT $54 / 9$ (6.04, 6.26 and 6.22 ton in plant cane, first ratoon and second ratoon, respectively). The highest RA value $(161.04 \%)$ for sugar yield was recorded by the genotype G 2008-11, indicating the superiority of sugar yield in second ratoon crop over plant cane crop, while the lowest RA value $(51.45 \%)$ for sugar yield was recorded by the genotype $\mathrm{G} 2008-50$, indicating the high reduction in yield in the second ratoon crop. It could be concluded that, among the evaluated Egypt. J. Agron. 37, No. 2 (2015) 
genotypes, the genotype G 2008-59 seems to be the ideal one because of its significant superiority in cane yield and its acceptable juice quality traits during the three crop cycles that resulted in higher average mean yield of sugar (6.45 ton/feddan) than that of the check cultivar GT 54/9 (6.17 ton/feddan). In general, the superiority of genotypes in sugar yields is firstly due to their superiority in cane yield. Milligan et al. (1990b), El- Hinnawy et al. (2001) and Masri et al. (2008) reported that cane yield was the predominant in determining sugar yield. Therefore, further improvement of sugar yield could be obtained through selection for high cane yield and its component traits.

Examination of variance components calculated from the full model analysis across crops (Tables 8,9) showed that the relative influence of genotypic variance $\left(\delta^{2} \mathrm{~g}\right)$ in determining the phenotypic variance was primary to genotype by crop interaction variance $\left(\delta^{2} \mathrm{gc}\right)$ for stalk length, stalk diameter, stalk weight, stalk density, stalks number, cane yield, sugar yield and juice quality traits. Error variance $\left(\delta^{2} \mathrm{e}\right)$ played a smaller role in influencing the phenotypic variance for all studied traits. Broad sense heritability $(\mathrm{H} \%)$ estimates were high for cane yield and its components as well as sugar yield, since it ranged from 82.53 for cane yield to 95.06 for stalk length, while it was high-moderate to moderate estimates for juice quality traits ( $59.26 \leq \mathrm{H} \% \geq 78.46$ ).

Genotypic coefficient of variation (GCV \%) facilitate comparisons among traits with different units and scales, and give perspective to the variability to be potentially exploited for genetic gain. High GCV estimates (Tables 8, 9) were for stalk weight (27.01), stalk density (39.36) and stalks number (32.57), moderate estimates were for stalk diameter (12.09), cane yield (16.12) and sugar yield (18.38), while low estimates were for stalk length and juice quality traits $(2.05 \leq$ GCV\% $\geq 7.99$ ).

TABLE 8. Variance components, mean, heritability (H\%), and genetic coefficient of variation (GCV\%) for stalk length, stalk diameter, stalk weight, stalk number and cane yield over crops.

\begin{tabular}{|c|c|c|c|c|c|c|}
\hline \multirow[b]{2}{*}{ Parameter } & \multicolumn{5}{|c|}{ Stalk } & \multirow[b]{2}{*}{$\begin{array}{c}\text { Cane yield } \\
\text { (ton/feddan) }\end{array}$} \\
\hline & $\begin{array}{c}\text { Length } \\
\text { (cm) }\end{array}$ & $\begin{array}{c}\text { Diameter } \\
(\mathbf{c m})\end{array}$ & $\begin{array}{c}\text { Weight } \\
\text { (kg) }\end{array}$ & $\begin{array}{l}\text { density } \\
\left(\mathrm{g} / \mathrm{cm}^{3}\right)\end{array}$ & $\begin{array}{c}\text { Number/ } \\
\text { feddan } x \\
10^{3}\end{array}$ & \\
\hline$\delta^{2} \mathrm{~g}$ & 455.501 & 0.085 & 0.06 & 0.144 & 242.527 & 40.159 \\
\hline$\delta^{2} \mathrm{gc}$ & 18.937 & 0.005 & 0.004 & 0.009 & 23.051 & 8.138 \\
\hline$\delta^{2} \mathrm{e}$ & 4.745 & 0.003 & 0.002 & 0.003 & 0.301 & 0.362 \\
\hline Mean & 267.22 & 2.41 & 0.91 & 0.96 & 47.81 & 39.33 \\
\hline $\mathrm{H} \%$ & 95.06 & 91.40 & 90.91 & 92.31 & 91.22 & 82.53 \\
\hline GCV \% & 7.99 & 12.09 & 27.00 & 39.36 & 32.57 & 16.12 \\
\hline
\end{tabular}

Egypt. J. Agron. 37, No.2 (2015) 
TABLE 9. Variance components, mean, heritability $(\mathrm{H} \%)$ and genetic coefficient of variation $(\mathrm{GCV} \%)$ for Brix, sucrose \%, purity \%, sugar recovery\%, and sugar yield over crops.

\begin{tabular}{|c|c|c|c|c|c|}
\hline \multirow{2}{*}{ Parameter } & Brix & Sucrose & Purity & \multicolumn{1}{c|}{$\begin{array}{c}\text { Sugar } \\
\text { recovery }\end{array}$} & \multirow{2}{*}{$\begin{array}{c}\text { Sugar yield } \\
\text { (ton/feddan) }\end{array}$} \\
\cline { 2 - 5 } & \multicolumn{5}{|c|}{$\%$} \\
\hline$\delta^{2} \mathrm{~g}$ & 0.192 & 0.414 & 2.731 & 0.295 & 0.678 \\
\hline$\delta^{2} \mathrm{gc}$ & 0.110 & 0.097 & 0.923 & 0.064 & 0.131 \\
\hline$\delta^{2} \mathrm{e}$ & 0.022 & 0.020 & 0.389 & 0.017 & 0.008 \\
\hline Mean & 21.38 & 17.24 & 80.63 & 11.38 & 4.48 \\
\hline H\% & 59.26 & 77.97 & 67.55 & 78.46 & 82.99 \\
\hline GCV \% & 2.05 & 3.73 & 2.05 & 4.77 & 18.38 \\
\hline
\end{tabular}

The GCV values estimated in this study suggest a selection to improve a particular crop's yield component such as stalk weight, stalk density and stalks number. The different potential improvement among traits results at least in part from selection program's methodology prior to this selection stage (Breaux, 1972). Selection program in Egypt tends to concentrate on sucrose quality, stalk length and stalk diameter in its early stages. Therefore, genetic variability for these traits may be limited (Gravois, 1988 and Milligan, 1988).

It could be argued that a crop like sugarcane in which a single superior genotype once identified can be multiplied clonally. Therefore, estimates of broad sense heritability are more relevant to the breeder than those of narrow sense heritability. The previous results indicated high heritability estimates for cane yield components, cane yield and sugar yield, while moderate to low estimates were for juice quality traits, since it ranged from 59.26 for Brix to 78.46 for sugar recovery. High heritability with high to moderate GCV was observed for stalk weight, stalk density, stalks number, cane yield, and sugar yield, suggesting the possibility of improvement of those traits through selection. Although heritability of stalks number, Brix, sucrose percentage, juice purity, and sugar recovery were relatively high, a lack of remaining variability at this stage of selection left little potential for more gain. In this study, estimates of heritability based on the full model analysis across crops because of estimates of heritability within a crop under one environment, somewhat consider biased estimates, where the environmental effects are known to be significant in sugarcane (Hogarth et al., 1981 and Schnell \& Nagai, 1992). Bias in heritabilities estimated under restricted environmental conditions was discussed by Dudley \& Moll (1969).

Egypt. J. Agron . 37, No. 2 (2015) 
Since the goals of increased ratooning ability (RA) and improved ratoon crops yields are similar, comparison with heritability and estimated G C V for selection in the ratoon crop are of interest. Genotypic variance $\left(\delta^{2} \mathrm{~g}\right)$ was primary to error variance $\left(\delta^{2} \mathrm{e}\right)$ for RA of cane yield and its components as well as sugar yield and juice quality traits (Tables, 10,11). The largest heritability estimates of RA were for stalk weight $(98.10 \%)$, stalk density $(98.24 \%)$, stalks number $(99.46 \%)$ and sugar yield with high genotypic coefficient of variation (GCV) estimates for the same traits $(22.78,32.26,36.41$ and 27.26 , respectively). The GCV estimates of RA were low for stalk length, stalk diameter, and cane yield, as well as, juice quality traits $(3.21 \% \leq \mathrm{G} \mathrm{C} \mathrm{V} \leq 10.05 \%)$. El-Hinnawy \& Masri (2009a) reported broad-sense heritability and GCV for the second ratoon crop stalk length, stalk weight, stalks number, cane yield and sugar yield to be less than or equal heritability and GCV of RA for these traits. Thus selection for high RA seems to offer considerable potential improvement for these traits.

TABLE 10. Variance components, means, heritability $(H)$ and genetic coefficient of variation (GCV) of ratooning ability for cane yield and its components in sugarcane.

\begin{tabular}{|c|c|c|c|c|c|c|}
\hline \multirow{2}{*}{ Parameter } & \multicolumn{5}{|c|}{ Stalk } & \multirow{2}{*}{$\begin{array}{c}\text { Cane } \\
\text { yield }\end{array}$} \\
\cline { 2 - 6 } & Length & Diameter & Weight & Density & Number & 19.726 \\
\hline$\delta^{2} \mathrm{~g}$ & 23.944 & 95.782 & 503.678 & 1037.02 & 1189.09 & 19.04 .52 \\
\hline$\delta^{2} \mathrm{e}$ & 3.293 & 4.332 & 9.734 & 18.526 & 6.403 & 4.396 \\
\hline Mean & 101.56 & 97.37 & 98.5 & 99.83 & 94.72 & 87.23 \\
\hline H\% & 87.91 & 95.67 & 98.10 & 98.24 & 99.46 & 81.78 \\
\hline GCV\% & 4.82 & 10.05 & 22.78 & 32.26 & 36.41 & 5.09 \\
\hline
\end{tabular}

TABLE 11. Variance components, means, heritability $(H)$ and genetic coefficient of variation (GCV) of ratooning ability for sugar yield and juice quality traits in sugarcane.

\begin{tabular}{|c|c|c|c|c|c|}
\hline \multirow{2}{*}{ Parameter } & \multicolumn{1}{|c|}{ Brix } & Sucrose & Purity & $\begin{array}{c}\text { Sugar } \\
\text { recovery }\end{array}$ & \multirow{2}{*}{ Sugar yield } \\
\cline { 2 - 5 } & \multicolumn{5}{|c|}{$\%$} \\
\hline$\delta^{2} \mathrm{~g}$ & 19.726 & 24.468 & 10.697 & 37.284 & 670.35 \\
\hline$\delta^{2} \mathrm{e}$ & 4.396 & 6.753 & 4.448 & 12.641 & 22.695 \\
\hline Mean & 105.53 & 107.40 & 101.85 & 108.58 & 94.98 \\
\hline H\% & 81.78 & 78.37 & 70.63 & 74.68 & 96.73 \\
\hline GCV\% & 4.21 & 4.61 & 3.21 & 5.62 & 27.26 \\
\hline
\end{tabular}




\section{References}

Association of Official Agricultural Chemists (AOAC) (1995) "Official Methods of Analysis" published by the A.O.A.C., Box540, Washington .USA.

Arain, M.Y., Panhwar, R.N., Gujar, N., Chohan, M., Rajput, M. A., Soomro, A. F. and Junejo, S. (2011) Evaluation of new candidate sugarcane varieties for some qualitative and quantitative traits under Thatta Agro-climatic conditions. The Journal of Animal and Plant Sciences, 21(2), 226-230.

Breaux, R.D. (1972) Selecting commercial sugarcane varieties from large seedling and clonal population. Proc. Am. Soc. Sugar Cane Technol. 2(ns), 58-66.

Bhatnagar, P.K., Khan, A.Q., Singh, A. and Khan, K.A. (2003) Studies on genetic variability, heritability and genetic advance in plant and ratoon crops of sugarcane. Indian Sugar. J. 111 (3), 183 - 185.

Chapman, L.S. (1988) Constraints to production in ratoon crops. Proc. Australian Soc. Sugar Cane Technol. 10, pp. 189-192.

Chapman, L.S., Ferraris, R. and Ludlow, M.M. (1992) Ratooning ability of cane varieties: Variation in yield and yield components. Proc. Australian Soc. Sugar Cane Technol. 14, pp. 130-138.

Dudley, J.W. and Moll, R.H. (1969) Interpretation and use of estimates of heritability and genetic variances in plant breeding. Crop Sci. 9, 257-262.

EL- Hinnawy, H.H. and Masri, M.I. (2009a) Crop cycle effects on genetic variability, heritability and yield of sugarcane. J. Agric. Sci. Mansoura Univ. 34 (6), 6755- 6767.

El-Hinnawy, H.H. and Masri, M.I. (2009 b) Ratooning ability and indirect response to selection in sugarcane. Egypt. J. Plant Breed. 13, 39-52.

El- Hinnawy, H.H., Allam, A.I., Mahmoud, E.A. and Masri, M.I. (2001) Crop effects on phenotypic and genotypic relationships among sugarcane traits. Proceedings of the $2^{\text {nd }}$ Congress of Plant Breeding, Assuit, Egypt, pp. $295-311$.

Falconer, D.S. (1989) "Introduction to Quantitative Genetics". $3^{\text {rd }}$ ed., Longman Scientific \& Technical, London.

Freed, R.S.P., Einensmith, S., Gutez, S., Reicosky, D., Smail, V.W. and Wolberg, P. (1989) "MSTAT-C Analysis of Agronomic Research Experiments". Michigan Univ. East Lansing, USA.

Gravois, K.A. (1988) Path-coefficient analysis. A study of phenotypic, broad-sense and narrow-sense variation in sugar cane . Ph D. Thesis. Louisiana State Univ., Baton Rouge ( Diss. Abstr. 89 - 17818).

Herbert, L.P. (1973) Testing of sugarcane varieties for milling quality. The Sugar J. 36(4), 8-12.

Egypt. J. Agron . 37, No. 2 (2015) 
Hogarth, D.M., Wu, K.K. and Heinz, D.J. (1981) Estimating genetic variance in sugarcane using factorial cross design. Crop Sci. 21, $21-25$.

Jamil, M. S., Afghan, S., Majid, M.A. and Rasool, A. (2007) Ratooning performance of different sugarcane varieties. Pakistan Sugar J. 22(3), 38- 47.

Jamoza, J.E., Owuoche, J., Kiplagat, O. and Opile, W. (2014) Broad- sense heritability estimation and correlation among sugarcane (Saccharum spp. hybrids) yield and some agronomic traits in western Kenya. Inter. J. Agric. Policy Res. 2(1),16-25.

Johnson, J.L., Heagler, A.M., Zapata, H.O. and Ricaud, R. (1993) The impact of succession planting and a third ratoon crop on economic efficiency in sugarcane production in Louisiana. Am. Soc. Sugarcane Technol. 13, 28-32.

Kang, M.S., Miller, J.D. and Tai, P.Y.P. (1983) Genetic and phenotypic path analysis and heritability in sugarcane. Crop Sci. 23, 643-647.

Kang, M.S., Miller, J.D. and Tai, P.Y.P. (1984) Clonal and individual repeatability of agronomic traits in sugarcane. Am. Soc. Sugarcane Technol. 3, 22-27.

Masri, M.I., Abd EL-Shafi, M.A. and EL-Taib, A.B.A. (2008) Trait relationships in sugarcane at final selection stages. Annals of Agric., Sci., Moshtohor, 46(4), $241-$ 253.

Mathur, R.B.L. (1997) "Handbook of Cane Technology". Oxford IBH Publishing Co. PVT.LTD. New Delhi. Calcutta.

Milligan, S.B. (1988) The genetic variance-covariance structure of a Louisiana sugarcane breeding population. Ph D. Thesis. Louisiana State Univ., Baton Rouge (Diss. Abstr. 88 - 19963).

Milligan, S.B., Gravois, K.A. and Martin, F.A. (1996) Inheritance of sugarcane ratooning ability and relationship of younger crop traits to older crop traits. Crop Sci. 36, $45-50$.

Milligan, S.B., Gravois, K.A., Bischoff, K.P. and Martin, F.A. (1990 a) Crop effects on broad-sense heritabilities and genetic variances of sugarcane yield component. Crop Sci. 30, $344-349$.

Milligin, S.B., Grvois, K.A., Bischoff, K.P. and Martin, F.A. (1990 b) Crop effects on genetic relationships among sugarcane traits. Crop Sci. 30, $927-931$.

Mirzawan, P.D.N. and Sugiyarta, E. (1999) Ratooning ability in sugarcane: Direct vs indirect selection based on clonal performance in younger crops. Proc. Int. Soc. Sugarcane Technol. 23, 467-475.

Olaoye, G. (2005) Estimate of ratooning ability in sugarcane (Saccharum officinarum L.) under conditions of low-available soil moisture in a savanna ecology of Nigeria. Moor Journal of Agricultural Research, 6 (1\&2),16-23. 
Orgeron, A.J., Gravois, K.A. and Bischoff, K.P. (2007) Planting rate effects on sugarcane yield trials. J. Amer. Soc. Technol. 27, 23 - 34.

Ricaud, R. and Arceneaux, A. (1986) Some factors affecting ratoon cane yield and longevity in Louisiana. Proc. Int. Sugar Cane Technol. 19, pp.18-24.

Salassi, M.E. and Giesler, G.G. (1995) Projected costs and returns-sugarcane, Louisiana, 1995. Dept. of Agric. Economics and Agribusiness, AEA Info. Series No. 132, LAES, LSU Agric. Ctr., Baton Rouge.

Schnell, R.J. and Nagai, C. (1992) Variation in agronomic characters among maternal half-sib families of Saccharum officinarum and elite Hawaiian commercial clones. Trop. Agric. 69, $203-206$.

Shrivastava, A.K., Ghosh, A.K. and Agnihotri, V.P. (1992) "Sugarcane Rations". Oxford \& IBH Publishing Co. Pvt. Ltd., New Delhi.

Steel, R.G.D., Torrie, J.H. and Dickey, D.A. (1997) "Principles and Procedures of Statistics: A Biometrical Approach", $3^{\text {rd }}$ ed,. Mc Graw-Hill, New York, 6666.

Tahir, M., Khalil, I.H., McCord, P.H. and Glaz, B. (2014) Sugarcane genotype performance in three environments (based on crop cycle) at Mardan, Pakistan. American Journal of Experimental Agriculture, 4(3), 362-375.

Tripathi, B.K., Gill, S.S., Misa, G.P. and Lai, S. (1982) Screening of sugarcane (Saccharum spp. hybrids) genotypes for ratooning ability. Indian Sugar, 32, 577-580.

Yadav, R.L. and Sharma, R.K. (1980) Effect of nitrogen levels and harvesting dates on quality characters and yield of four sugar cane genotypes. Indian Journal, 50(7), 581 589.

(Received 18/5/2015; accepted $18 / 8 / 2015)$ 


\title{
المحصول والقدرة على التظليف لثلاثين تركيب وراثي من قصب

\author{
محمد إبراهيم مصري و محمد مصطفى محمد أمين

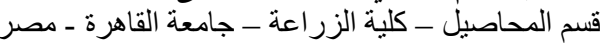

أجريت تجربة حقلية بمحطة بحوث المطاعنة بمحافظة الأقصر وذللك بهدف تقبيم

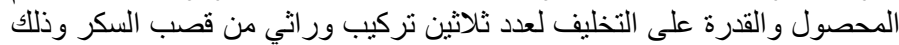

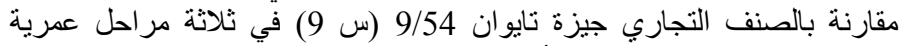

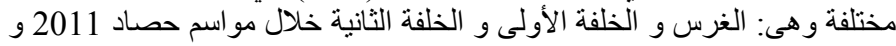
2012 و 2013. تم استخدام تصميم القطاعات الكاملة العشو ائية مع ثلاثة مكررات

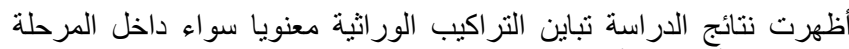
العمرية الواحدة أو بين الأعمار الثثلاثة وذللك بالنسبة لجميع الصفات التهات تحت الدان الدراسة

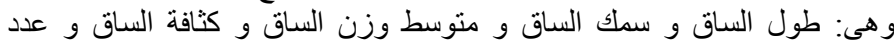

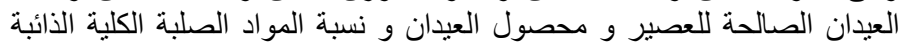

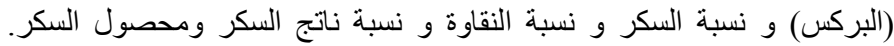

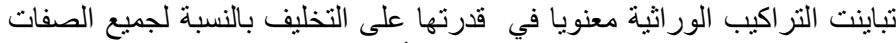

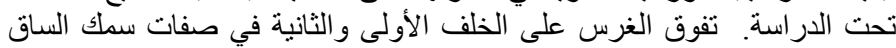

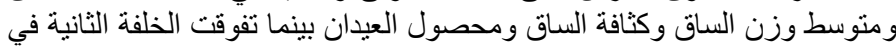

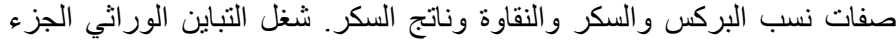

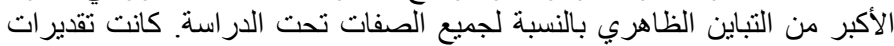

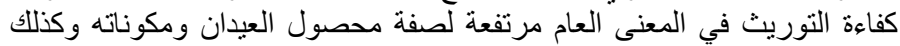

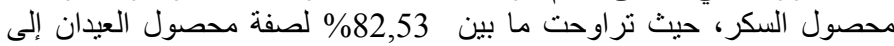

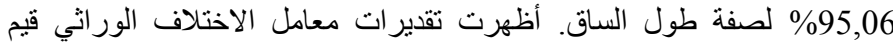

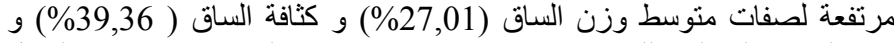

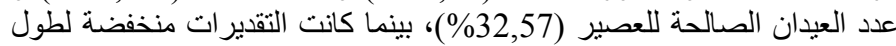

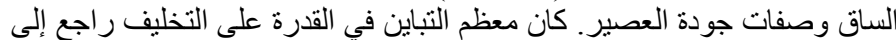

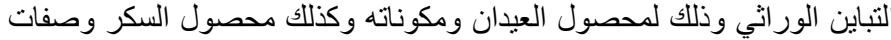

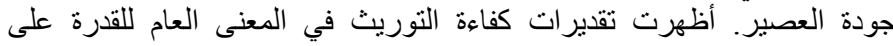

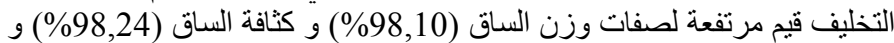

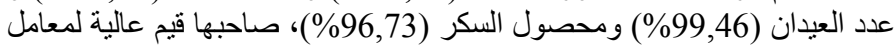
الاختلاف الور اني لنفس الصفات السابقة (22,78\% ، 32,26\% ، 36,41\% و

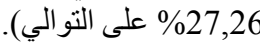

